\title{
Correction: The Rho/ROCK pathway for lysophosphatidic acid- induced proteolytic enzyme expression and ovarian cancer cell invasion
}

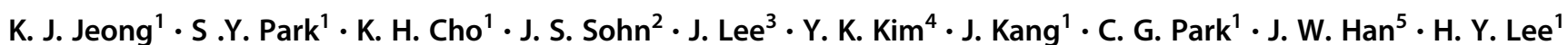

Published online: 29 March 2019

(c) Springer Nature Limited 2019

Correction to: Oncogene (2012) 31:4279-4289

https://doi.org/10.1038/onc.2011.595

published online 16 January 2012

The original version of this article contained an error in the published figures Fig. 2 and Fig. 3f, where the information was inadvertently duplicated. This error does not alter the conclusions of the paper. The corrected figures are published in this correction notice. The authors sincerely apologize for this error.

H. Y. Lee

hoi@konyang.ac.kr

1 Department of Pharmacology, Myunggok Medical Research Institute, College of Medicine, Konyang University, Daejeon, Korea

2 Department of Pathology, Myunggok Medical Research Institute, College of Medicine, Konyang University, Daejeon, Korea

3 Department of Microbiology, Myunggok Medical Research Institute, College of Medicine, Konyang University,

Daejeon, Korea

4 Department of Pharmacology, College of Pharmacy, Sookmyung Women's University, Seoul, Korea

5 Department of Biochemistry and Molecular Biology, School of Pharmacy, Sungkyunkwan University, Suwon, Korea 
Fig. 2 MMP-9 and uPA are critical for ovarian cancer cell invasion. The cells were transfected with indicated siRNA and then stimulated with or without LPA for $24 \mathrm{~h}$. Invading cells captured by microscope (top). Invasion assay by counting invading cells (bottom; $* P<0.05$ versus control, ${ }^{\#} P<0.05$ versus LPA treatment without siRNA transfection). Error bars, s.d., analysis of variance. All experiments were repeated three times

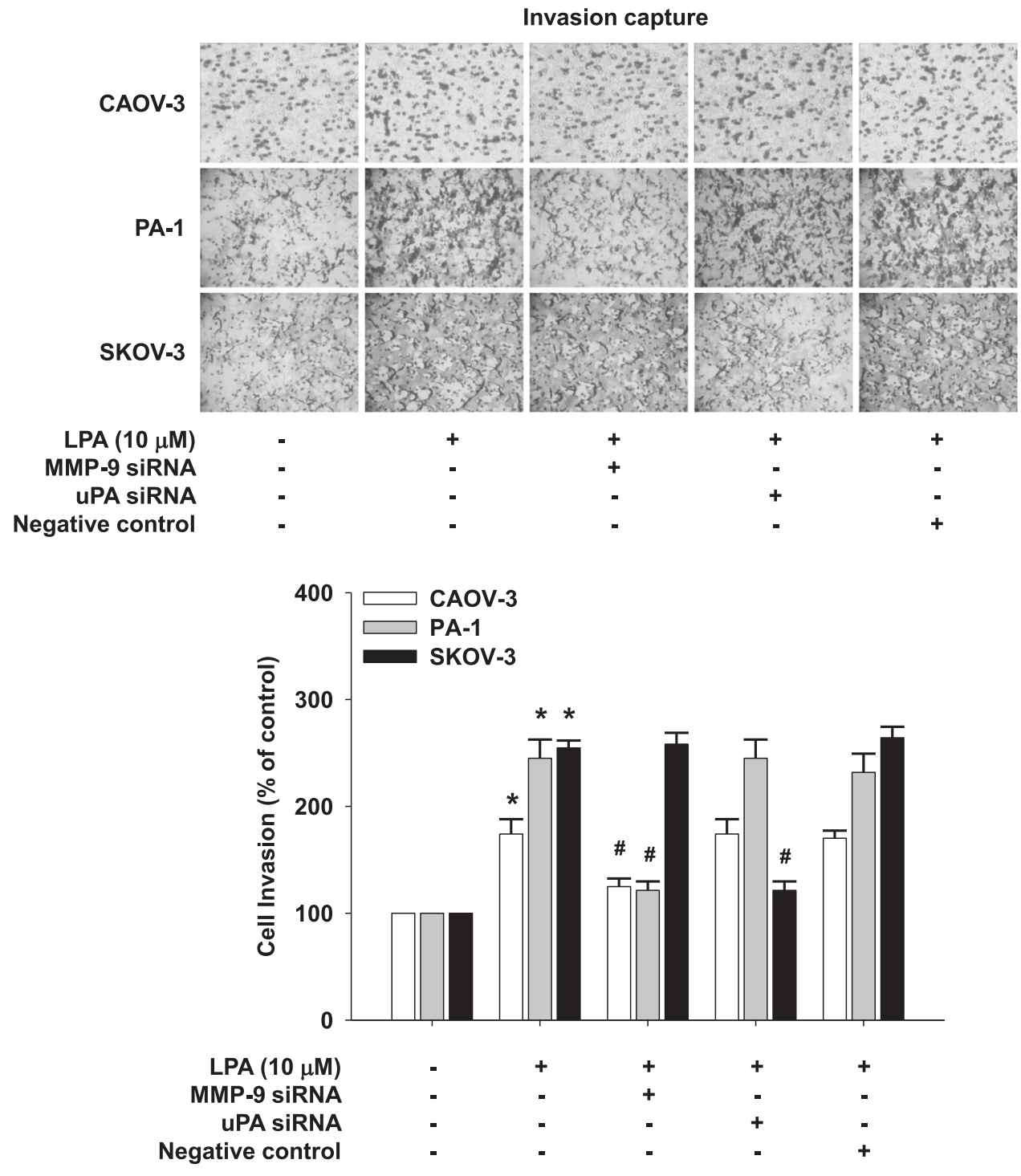


f

Motility capture
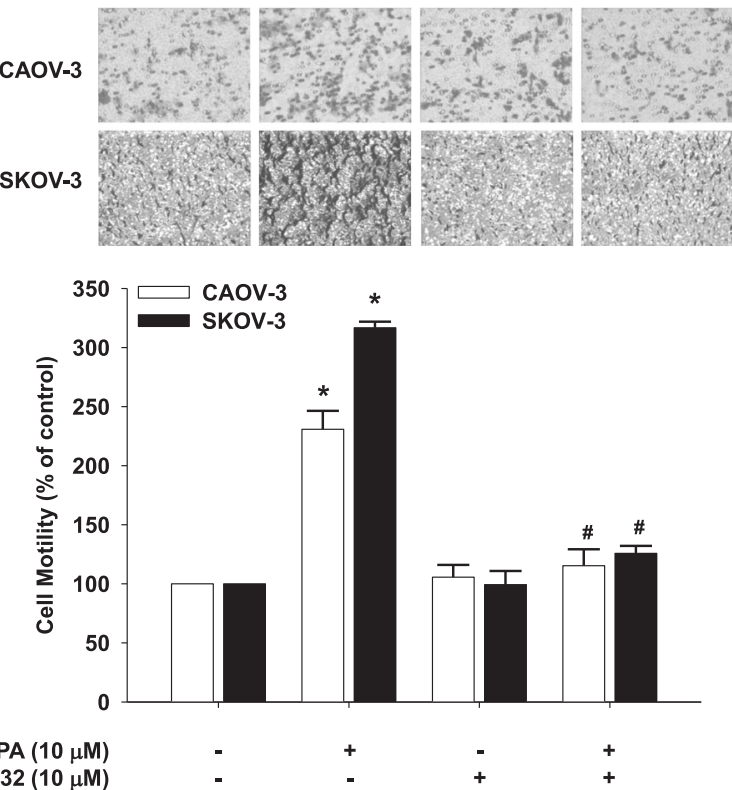

Fig. 3 f Cell migration assay by modified Boyden chamber $(* P<0.05$ versus control, ${ }^{\sharp} P<0.05$ versus LPA treatment only) 